\title{
Scaling regimes for second layer nucleation
}

\author{
Joachim Krug \\ Fachbereich Physik, Universität GH Essen, D-45117 Essen, Germany
}

(October 31, 2018)

\begin{abstract}
Nucleation on top of two-dimensional islands with step edge barriers is investigated using scaling arguments. The nucleation rate is expressed in terms of three basic time scales: The time interval between deposition events, the residence time of atoms on the island, and the encounter time required for $i^{*}+1$ atoms forming a stable nucleus to meet. Application to the problem of second-layer nucleation on growing first layer islands yields a sequence of scaling regimes with different scaling exponents relating the critical island size, at which nucleation takes place, to the diffusion and deposition rates. Second layer nucleation is fluctuation-dominated, in the sense that the typical number of atoms on the island is small compared to $i^{*}+1$, when the first layer island density exponent $\chi$ satisfies $\chi\left(i^{*}+1\right)<2$. The upper critical nucleus size, above which the conventional mean-field theory of second layer nucleation is valid, increases with decreasing dimensionality. In the related case of nucleation on top of multilayer mounds fluctuation-dominated and mean-field like regimes coexist for arbitrary values of the critical nucleus size $i^{*}$.
\end{abstract}

PACS: 68.35.Fx, 81.15.Aa, 05.40.-a

\section{INTRODUCTION}

Atomistic nucleation theory was developed in the sixties and seventies in response to the realization that critical nuclei of atomic dimensions are common in far from equilibrium thin film growth [1 3]. During the past decade the subject has experienced a vigorous renaissance, driven by the invention of atomic scale imaging techniques as well as by the availability of realistic large scale simulations [ $[$. While this has resulted in a wide range of new theoretical developments, the classical theory has stood the test of time remarkably well. This is surprising because classical nucleation theory relies, in the jargon of statistical mechanics, on an approximation of mean field type, in the sense that it considers only the evolution of spatially averaged concentrations of the different surface species - adatoms and clusters - and thereby neglects effects of spatial correlations and fluctuations.

It was recently pointed out that the classical theory manifestly fails in describing nucleation events on top of islands bounded by strong step edge barriers [5.66. The onset of second layer nucleation on top of the first layer islands is an important factor determining the growth mode and morphology of multilayer films. Tersoff and coworkers [7] developed a theory of second layer nucleation based on the classical mean field estimate

$$
\omega_{\mathrm{mf}} \sim \nu A n^{i^{*}+1}
$$

for the rate $\omega$ of nucleation events on the island, in terms of the in-layer hopping rate $\nu$, the island area $A$, the adatom density $n$ and the size $i^{*}$ of the critical (= largest unstable) cluster. In Refs. 同, 6 it was shown that for $i^{*}=1,2$ the mean field estimate disagrees with microscopic considerations 8 10] and computer simulations. The failure of mean field theory was qualitatively attributed to the small number of atoms typically present on the island, and the associated large fluctuations in the occupancy of the island.

The question then arises whether mean field behavior may be recovered at larger values of the critical nucleus size, where nucleation necessarily involves a greater number of atoms, and thus fluctuation effects should be reduced. A partial answer to this question was provided in recent work by Heinrichs, Rottler and Maass (HRM) [11. They distinguish between two different nucleation modes: In the fluctuation-dominated mode the mean number of adatoms on the island (in the quasi-stationary state prior to nucleation) is much smaller than the number $i^{*}+1$ required for nucleation, and therefore nucleation is a rare, large fluctuation, while in the mean field regime, which corresponds to the classical approach of [7], the mean number of adatoms exceeds $i^{*}+1$. Consistency considerations then show that second layer nucleation on top of first layer islands is fluctuation-dominated for $i^{*} \leq 2$ and mean-field-like for $i^{*} \geq 3$.

In this paper I elaborate on the observations of HRM, with the goal of further clarifying the role of fluctuations in second layer nucleation. I begin by rederiving the main results of HRM within the approach of [6], which relies on expressing the nucleation rate in terms of the basic time scales of the process. While less quantitative than the treatment of [1], this provides, perhaps, a somewhat more transparent and unified view. A simple, universal criterion for fluctuation-dominated second layer nucleation is found which involves the first layer island density exponent and the critical nucleus size (Eq.(28)). I then extend the analysis to $d$-dimensional nucleation, finding the same general structure as in the two-dimensional case, but with a decreasing influence of fluctuations with increasing $d$; as a byproduct, I recover the expression for the one-dimensional first layer island density exponent recently derived in Ref. 
[12]. Finally, I generalize the theory of nucleation on top of multilayer mounds developed in [6] to $i^{*}>1$. In contrast to the case of nucleation on first layer islands, here fluctuation-dominated and mean field like nucleation regimes are found to coexist for arbitrary values of $i^{*}$. This illustrates that the importance of fluctuations depends crucially on the growth situation.

\section{STATIONARY RATES OF FLUCTUATION-DOMINATED NUCLEATION}

We consider a two-dimensional island of fixed linear extension $L$. Atoms are deposited onto the island at rate $F$, they migrate on the island with an in-layer hopping rate $\nu$ and descend from the island at the inter-layer hopping rate $\nu^{\prime}$. The mean time interval between subsequent arrivals of atoms on the island is then

$$
\Delta t \sim \frac{1}{F L^{2}}
$$

and the mean residence time of an atom on the island in steady state is of the form [5,6, 11]

$$
\tau=\frac{a L^{2}}{\nu}+\frac{b L}{\nu^{\prime}}
$$

where $a$ and $b$ are geometry-dependent constants. The first term on the right hand side of (3) is of the order of the traversal time $\tau_{\operatorname{tr}} \sim L^{2} / \nu$ required for the atom to migrate across the island, while the second term describes the increase in the residence time due to the step edge barriers. The second term dominates if $L \ll l_{\mathrm{ES}}$, where

$$
l_{\mathrm{ES}} \approx \nu / \nu^{\prime}
$$

is the Ehrlich-Schwoebel length [9].

The stationary mean number of atoms on the island is given by $\tau / \Delta t$ 6.11], and hence the condition for the fluctuation-dominated mode of HRM reads

$$
\frac{\tau}{\Delta t} \ll 1
$$

It will turn out to be useful to write this in the form $L \ll L_{F}$, where the flux scale $L_{F}$ is given by

$$
L_{F} \sim \begin{cases}\left(\nu^{\prime} / F\right)^{1 / 3} & : \quad L \ll l_{\mathrm{ES}} \\ (\nu / F)^{1 / 4} & : \quad L \gg l_{\mathrm{ES}} .\end{cases}
$$

For given $L, \nu$ and $\nu^{\prime}$, Eq.(5) can always be satisfied by making the flux sufficiently small.

Nucleation with a critical nucleus size $i^{*}$ is treated within the "noninteracting particle model" [11], in which the lifetimes of all unstable clusters are assumed comparable to the inverse hopping rate $1 / \nu$. Then the third relevant time scale in the problem, in addition to (2) and (3), is given by the encounter time $\tau_{\text {enc }}$ required for the $i^{*}+1$ atoms to find each other on the island. In order of magnitude we have 11

$$
\tau_{\text {enc }} \sim L^{2 i^{*}} / \nu
$$

A simple derivation is given below in Section IV.

Under the condition (5), each nucleation event is uniquely associated with the deposition of the $i^{*}+1^{\prime}$ th atom. The number of nucleation events per unit time can then be written as

$$
\omega=p_{\text {nuc }} / \Delta t
$$

where $p_{\text {nuc }}$ is the probability for a freshly deposited atom to participate in a nucleation event. The latter is generally of the form

$$
p_{\text {nuc }}=p_{i^{*}+1} \cdot p_{\mathrm{enc}},
$$

where $p_{i^{*}+1}$ denotes the probability that $i^{*}$ atoms were present on the island prior to the arrival of the $i^{*}+1^{\prime}$ th, and $p_{\text {enc }}$ is the probability that the $i^{*}+1$ atoms encounter each other before one of them leaves the island again. The quantity $p_{i^{*}+1}$ is given by [6] 


$$
p_{i^{*}+1}=\frac{1}{i^{*} !}\left(\frac{\tau}{\Delta t}\right)^{i^{*}}
$$

The encounter probability can be estimated as 11]

$$
p_{\mathrm{enc}} \approx 1-e^{-\tau / \tau_{\mathrm{enc}}}
$$

where for simplicity we have equated the residence time of the $i^{*}+1$ atoms with that of a single atom (in fact the two differ by a factor $\left.i^{*}+1\right)$. Thus $p_{\text {enc }}$ is of order unity for $\tau / \tau_{\text {enc }} \gg 1$ and of the order $p_{\text {enc }} \approx \tau / \tau_{\text {enc }}$ when $\tau / \tau_{\text {enc }} \ll 1$.

In the case $i^{*}=1$ the encounter time (7) is of the same order as the traversal time $L^{2} / \nu$, whereas the residence time (3) is of the order of $\tau_{\mathrm{tr}}$ or larger. Thus one always has $p_{\text {enc }}=\mathcal{O}(1)$ and $p_{\text {nuc }} \approx p_{2}$ [6]. In contrast, for $i^{*}>1$ it is possible to have a transition from $\tau \gg \tau_{\text {enc }}$ to $\tau \ll \tau_{\text {enc }}$ with increasing island size. This obviously requires that $\nu / \nu^{\prime} \gg 1$, so that $\tau \sim L / \nu^{\prime} \gg \tau_{\text {enc }}$ for small islands, and occurs at the characteristic length scale

$$
L_{\times} \sim\left(\nu / \nu^{\prime}\right)^{1 /\left(2 i^{*}-1\right)}
$$

We note that this is small compared to the Ehrlich-Schwoebel length, and therefore the transition to encounter-limited nucleation occurs before the step edge barriers become irrelevant, in the sense that $\tau$ becomes of the same order as $\tau_{\operatorname{tr}}$.

In the encounter-limited regime the expression (8) agrees in order of magnitude with the mean field estimate (1). To see this, note that the adatom density on the island is of order $\tau /\left(\Delta t L^{2}\right)=F \tau$, and hence

$$
\omega_{\mathrm{mf}} \sim \nu L^{2}\left(\frac{\tau}{L^{2} \Delta t}\right)^{i^{*}+1} \approx \frac{1}{\Delta t}\left(\frac{\tau}{\Delta t}\right)^{i^{*}} \frac{\tau}{\tau_{\mathrm{enc}}} \approx \frac{p_{i^{*}+1} \cdot p_{\mathrm{enc}}}{\Delta t} .
$$

In this sense the mean field approach remains applicable also outside of its strict range of validity 11 .

We summarize the preceding considerations in the following three expressions for the fluctuation-dominated nucleation rate, which apply with increasing island size $L$ :

$$
\begin{array}{cc}
\omega \approx \frac{1}{\Delta t}\left(\frac{\tau}{\Delta t}\right)^{i^{*}} \sim F\left(F / \nu^{\prime}\right)^{i^{*}} L^{3 i^{*}+2} & L \ll L_{\times} \quad(\text { regime II }) \\
\omega \approx \frac{1}{\tau_{\text {enc }}}\left(\frac{\tau}{\Delta t}\right)^{i^{*}+1} \sim F\left(F / \nu^{\prime}\right)^{i^{*}}\left(\nu / \nu^{\prime}\right) L^{i^{*}+3} & L_{\times} \ll L \ll l_{\mathrm{ES}} \quad \text { (regime III) } \\
\omega \approx \frac{1}{\tau_{\mathrm{enc}}}\left(\frac{\tau}{\Delta t}\right)^{i^{*}+1} \sim F(F / \nu)^{i^{*}} L^{2 i^{*}+4} & L \gg l_{\mathrm{ES}} \quad \text { (regime IV). }
\end{array}
$$

Regime III does not exist for $i^{*}=1$. For $i^{*}>1$, the encounter-limited regimes III and IV extend also into the mean field realm, while regime II occurs only in the fluctuation-dominated nucleation mode.

Note that Eqs.(14) and (15) correspond to the regimes II and I identified in [6] for $i^{*}=2$. Here we prefer to enumerate the regimes in accordance with HRM [11]. Their regime I will be dealt with below in Section IIIB.

\section{CONSISTENT SCALING REGIMES IN TWO DIMENSIONS}

While Eqs. 14, 15, 16) provide, in the specified ranges of parameters, valid expressions for the nucleation rate on top of an island of given size, in their application to a specific growth situation additional consistency requirements arise [11]: When a given expression for $\omega$ is used to compute the island size at which nucleation occurs, it has to be checked that this island size does indeed lie in the appropriate range.

In this section we derive the resulting scaling regimes for second layer nucleation in two dimensions. Following earlier work 5 76, we assume a population of equal sized first layer islands of density $N$. The island size increases with time $t$ or coverage $\theta=F t$ according to the growth law

$$
L(t) \sim \sqrt{\theta / N}
$$

For a nucleation rate of the form

$$
\omega=F \Omega L^{k}
$$


the critical island size at which a second layer nucleus forms is then given by [6]

$$
L_{c} \sim(N \Omega)^{-1 /(k+2)} .
$$

We further introduce the notation [1]

$$
\Gamma \equiv \nu / F, \quad \alpha \equiv \nu^{\prime} / \nu=l_{\mathrm{ES}}^{-1},
$$

where usually $\Gamma \gg 1, \alpha \ll 1$. The first layer island density is then of the order of

$$
N \sim \Gamma^{-\chi}
$$

which defines the island density exponent $\chi$. In standard nucleation theory [3] it is given by the expression

$$
\chi=\frac{i^{*}}{i^{*}+2} .
$$

At the moment we will however leave $\chi$ unspecified to allow for the possibility of different first layer nucleation mechanisms. Generalizations of (22) will be derived in Section IV.

\section{A. Stationary nucleation regimes}

Inserting Eqs.(14,15,16) into (19) one obtains expressions for the critical island size of the general form [11

$$
L_{c} \sim \Gamma^{\gamma} \alpha^{\mu}
$$

where the exponents $\mu$ and $\gamma$ are given by

$$
\begin{gathered}
\gamma=\frac{\chi+i^{*}}{3 i^{*}+4}, \quad \mu=\frac{i^{*}}{3 i^{*}+4} \quad \text { (regime II) } \\
\gamma=\frac{\chi+i^{*}}{i^{*}+5}, \quad \mu=\frac{i^{*}+1}{i^{*}+5} \quad \text { (regime III) } \\
\gamma=\frac{\chi+i^{*}}{2 i^{*}+6}, \quad \mu=0 \quad(\text { regime IV }) .
\end{gathered}
$$

Consider first regime II. Consistency requires the inequalities $L_{c} \sim \alpha^{\mu} \Gamma^{\gamma} \ll L_{\times} \sim \alpha^{-1 /\left(2 i^{*}-1\right)}$ and $\alpha^{\mu} \Gamma^{\gamma} \ll L_{F} \sim$ $(\alpha \Gamma)^{1 / 3}$ to be satisfied simultaneously, which implies

$$
\Gamma^{-(1-3 \gamma) /(1-3 \mu)} \ll \alpha \ll \Gamma^{-\gamma /\left(\mu+1 /\left(2 i^{*}-1\right)\right)} .
$$

Since $\alpha \ll 1$ and $\Gamma \gg 1$, these inequalities can be satisfied only if the exponent of $\Gamma$ on the left hand side is smaller than the one on the right hand side. Inserting the expressions (24) this is found to lead to the condition

$$
\chi<\frac{2}{i^{*}+1} .
$$

The analysis of regime III, based on the requirement $L_{\times} \ll L_{c} \ll L_{F}$, yields the same condition (28), while in regime IV the relevant inequalities $l_{\mathrm{ES}} \ll L_{c} \ll L_{F}$ translate into

$$
\chi<\frac{3-i^{*}}{2}
$$

Using the expression (22) for $\chi$, we see that the fluctuation-dominated regimes II and III become inconsistent at $i^{*}=i_{c 1}^{*}=(1+\sqrt{17}) / 2 \approx 2.56$. For larger $i^{*}$ regime III survives in its mean field version, while regime II disappears. In regime IV the transition from fluctuation-dominated to mean-field behavior occurs at $i^{*}=i_{c 2}^{*}=2$; in this regime the critical island size is of the order of the first layer island distance, $\gamma=\chi / 2$, and hence the problem really reduces to that of first layer nucleation.

The general form of the conditions (28,29) illustrates that the upper critical nucleus size, at which the mean-field nucleation mode sets it, is intimately linked to the expression for the first layer island density exponent. Scenarios different from the standard case (22) can arise if, due to e.g. metastable clusters [6] or surface reconstruction [13], the critical nucleus on the substrate is smaller or larger than that on the island. Another example of nonstandard behavior is kinetically limited first layer nucleation, where Eq.(22) is replaced by $14=2 i^{*} /\left(i^{*}+3\right)$ and the upper critical nucleus sizes shift to $i_{c 1}^{*}=\sqrt{3} \approx 1.732$ and $i_{c 2}^{*}=\sqrt{13}-2 \approx 1.6055$. In addition the growth law $(17)$ plays an important role, as will be discussed below. 


\section{B. Nonstationary nucleation}

For very strong step edge barriers the residence time $\tau$ becomes the largest time scale in the problem, and may effectively be set to infinity [6,11]. In this situation all atoms deposited on the island remain there, and a stationary state where deposition and loss of adatoms compensate each other is never reached. A distinction between fluctuationdominated and mean-field nucleation scenarios is nevertheless possible [11]: In the first case nucleation occurs as soon as the $i^{*}+1$-th atom arrives on the island, while in the second case many more atoms have to be deposited.

Using the growth law (17) it is easy to show that the time required to deposit a few (i.e., $\left.i^{*}+1\right)$ atoms on the island is

$$
\tau_{\text {dep }} \sim N^{1 / 2} / F
$$

At this point the island size is of the order $N^{-1 / 4}$, corresponding to exponents

$$
\gamma=\chi / 4, \quad \mu=0, \quad(\text { regime } \mathrm{I} / \mathrm{fl})
$$

for the fluctuation-dominated part of regime I. To see whether nucleation is actually fluctuation dominated, the time scale (30) is to be compared to the encounter time (7), evaluated at the island size $N^{-1 / 4}$; fluctuation-dominated nucleation occurs if $\tau_{\text {enc }} \ll \tau_{\text {dep }}$. Once more this yields the condition (28), which can therefore be regarded as a universal criterion for fluctuation-dominated second layer nucleation. For $\chi>2 /\left(i^{*}+1\right)$ mean field theory can be used to compute the nucleation rate, and one arrives at the scaling exponents 11$]$

$$
\left.\gamma=\frac{\chi\left(i^{*}+2\right)-1}{2 i^{*}+6}, \quad \mu=0 \quad \text { (regime } \mathrm{I} / \mathrm{mf}\right)
$$

A graphical representation of the sequence of scaling regimes as a function of $i^{*}$ is provided in Figure 1 .

While in the stationary nucleation regimes of Section III A the criterion (28) appears in a rather indirect way, in the nonstationary case it has a straightforward interpretation in terms of a comparison of the time scales $\tau_{\text {dep }}$ and $\tau_{\text {enc }}$. This is easily generalized to first layer island growth laws of the form

$$
L(t) \sim N^{-1 / 2} \theta^{\beta}
$$

which lead to

$$
\chi<\frac{2 \beta+1}{i^{*}+1}
$$

instead of (28).

\section{DIMENSIONALITY DEPENDENCE}

In statistical physics fluctuation effects are typically more prominent in low spatial dimensionalities. It is therefore of some interest to repeat the above considerations for growth of $d$-dimensional islands on a $d$-dimensional substrate. The residence time is then still given by an expression of the form (3), while the interarrival time becomes

$$
\Delta t \sim \frac{1}{F L^{d}}
$$

To estimate the encounter time, it is useful to visualize the trajectories of the $i^{*}+1$ diffusing adatoms in a configuration space of dimensionality $d\left(i^{*}+1\right)$, where the motion is restricted to a region of linear extent $L$. Nucleation occurs on a $d$-dimensional submanifold, the codimension of which is $d i^{*}$. We are thus dealing with a first passage problem in $d i^{*}$ dimensions, where the encounter time plays the role of the mean first passage time. This implies that

$$
\tau_{\text {enc }} \sim\left\{\begin{array}{lll}
L^{2} / \nu & : & d i^{*}<2 \\
L^{d i} / \nu & : & d i^{*} \geq 2
\end{array}\right.
$$

Since the residence time is always at least equal to the traversal time $L^{2} / \nu$, it follows that encounter-limited nucleation in the sense of $\tau_{\text {enc }} \gg \tau$ is possible only for dimensionalities $d>2 / i^{*}$. This recovers the upper critical dimension $d_{c}=2 / i^{*}$ for diffusion-limited reactions of $i^{*}+1$ particles [12, 15 . 


\section{A. Island density exponent in $d$ dimensions}

As a warm-up excercise we derive here a formula for the exponent $\chi$ of the first layer island density valid for general $d$. The appropriate expression for the nucleation rate is $(16)$, which is now to be interpreted as the rate of nucleation in a region of size $L$. The distance $l_{D} \sim N^{-1 / d}$ between first layer nuclei is determined by the condition that there should be one nucleation event per area $l_{D}^{d}$ during the monolayer deposition time $1 / F$, i.e.

$$
\omega\left(l_{D}\right) / F=\mathcal{O}(1)
$$

For $d i^{*} \geq 2$ this yields

$$
\chi=\frac{d i^{*}}{d+2+2 i^{*}} \quad\left(d i^{*} \geq 2\right)
$$

which agrees with (22) for $d=2$ and with the expression $\chi=i^{*} /\left(3+2 i^{*}\right)$ derived by Kallabis, Krapivsky and Wolf for $d=1, i^{*} \geq 2$ 12. For $d i^{*}<2$ we obtain instead

$$
\chi=\frac{d i^{*}}{(d+2) i^{*}+d} \quad\left(d i^{*}<2\right) .
$$

This case is physically realized only for $d=i^{*}=1$, where $(39)$ reduces to the well-known value $\chi=1 / 4$ 12, 16 .

These results are easily generalized to the growth of islands of (fractal or integer) dimensionality $d^{\prime}$ on a $d$ dimensional substrate. In that case the time of interest is not the monolayer growth time $1 / F$, but rather the time required to cover a fraction of the substrate which is of order unity. While the true coverage is related to island density and island size through $\theta \sim N L^{d^{\prime}}$, the covered fraction of the $d$-dimensional substrate is $\theta_{d} \sim N L^{d} \sim N^{1-d / d^{\prime}} \theta^{d / d^{\prime}}$. Setting the inverse nucleation rate equal to the time in which $\theta_{d}=\mathcal{O}(1)$ is reached then yields the condition

$$
\omega\left(l_{D}\right) / F \sim l_{D}^{d-d^{\prime}}
$$

which for $d i^{*} \geq 2$ implies the island density exponent

$$
\chi=\frac{d i^{*}}{2\left(i^{*}+1\right)+d^{\prime}} \quad\left(d i^{*} \geq 2\right) .
$$

For $d=1,2$ this agrees with the general expression given in [12].

\section{B. Second layer nucleation in $d$ dimensions}

For compact $d$-dimensional first layer islands the growth law (17) generalizes to

$$
L(t) \sim(\theta / N)^{1 / d},
$$

and the critical island size for a nucleation rate of the form (18) becomes

$$
L_{c} \sim(\Omega N)^{-1 /(d+k)} .
$$

Using the expressions (14,15,16) for the nucleation rates together with (35, 36) and (42) it is then straightforward to repeat the considerations of Section III. Rather than developing in detail the general case, we focus here on the transition between fluctuation-dominated and mean field nucleation regimes.

The analysis is most transparent in the nonstationary case. One finds that the deposition time is given by (30) for all $d$, and the critical island size for second layer nucleation is

$$
L_{c} \sim N^{-1 / 2 d} \sim \sqrt{l_{D}} \sim \Gamma^{\chi / 2 d} \quad(\text { regime I/fl). }
$$

Using this to evaluate the encounter time (7), it turns out that nucleation is always fluctuation-dominated for $d i^{*}<2$, while for $d i^{*} \geq 2$ the condition for the fluctuation-dominated regime is again given by the universal relation (28). The same relation also governs the disappearance of the fluctuation-dominated regime II for general $d$.

Setting the island density exponent (38) equal to $2 /\left(i^{*}+1\right)$ then yields the expression 


$$
i_{c 1}^{*}=\frac{1}{2 d}\left(4-d+\sqrt{8 d^{2}+(d+4)^{2}}\right)
$$

for the upper critical nucleus size at which the fluctuation-dominated regimes I/fl, II and III/fl disappear. This is a decreasing function of $d$ which approaches $i_{c 1}^{*}=1$ for $d \rightarrow \infty$. As expected, the fluctuation-dominated regime becomes smaller in higher dimensions.

To analyse the transition to mean field nucleation in regime IV we use the fact that the critical island size for second layer nucleation is of the order of the first layer island spacing, i.e. $\gamma=\chi / d$ and $L_{c} \sim l_{D}$. The condition for fluctuation-dominated nucleation therefore simply reads $\tau / \Delta t \sim F l_{D}^{2+d} / \nu \ll 1$, or $\chi<d /(2+d)$. This clearly always holds for the expression (39), while for (38) it is true provided $i^{*}<i_{c 2}^{*}$ with

$$
i_{c 2}^{*}=1+2 / d .
$$

Again $i_{c 2}^{*} \rightarrow 1$ for $d \rightarrow \infty$, and $i_{c 2}^{*}<i_{c 1}^{*}$ for all $d$.

The expressions for the scaling exponents $\gamma$ and $\mu$ in the remaining regimes read

$$
\begin{gathered}
\gamma=\frac{\chi\left(i^{*}+2\right)-1}{d\left(i^{*}+3\right)}, \quad \mu=0 \quad(\text { regime } \mathrm{I} / \mathrm{mf}) \\
\gamma=\frac{\chi+i^{*}}{(d+1) i^{*}+2 d}, \quad \mu=\frac{i^{*}}{(d+1) i^{*}+2 d} \quad \text { (regime II) } \\
\gamma=\frac{\chi+i^{*}}{i^{*}+2 d+1}, \quad \mu=\frac{i^{*}+1}{i^{*}+2 d+1} \quad \text { (regime III). }
\end{gathered}
$$

The scaling regimes for the one-dimensional case are shown in Figure 2 . The upper critical nucleus sizes are $i_{c 1}^{*}=$ $(3+\sqrt{33}) / 2 \approx 4.372 \ldots$ and $i_{c 2}^{*}=3$.

\section{NUCLEATION ON THE TOP TERRACE OF A MOUND}

In multilayer growth, the suppression of interlayer transport leads to the formation of pyramidal mounds [17]. For strong step edge barriers, in the sense that $l_{\mathrm{ES}}$ exceeds the distance $l_{D}$ between first layer islands, the mound separation is set by $l_{D}$ and remains constant during growth [18,19]. In this growth regime the mounds are weddingcake-like stacks of islands upon islands, with a characteristic up-down-asymmetry: While the valleys between mounds are deep crevices, at the hilltops flat terraces of a characteristic size $L_{\mathrm{top}}<l_{D}$ are found $10,19 . L_{\mathrm{top}}$ is determined by the nucleation rate on the top terrace through the requirement that on average one nucleation event should occur during the growth of a monolayer, so that [6,10]

$$
\omega\left(L_{\text {top }}\right) / F=\mathcal{O}(1) .
$$

For a nucleation rate of the general form (18) this implies

$$
L_{\text {top }} \sim \Omega^{1 / k} .
$$

A more precise calculation the factor of proportionality can be found in $[6]$.

As in the case of second layer nucleation, application of the expressions (14, 15, 16) for the nucleation rate yields estimates for the top terrace size of the form

$$
L_{\text {top }} \sim \Gamma^{\gamma^{\prime}} \alpha^{\mu^{\prime}}
$$

where the scaling exponents $\gamma^{\prime}$ and $\mu^{\prime}$ depend on $i^{*}$ and on the scaling regime in question. Inspection shows that the equivalent of the nonstationary regime I discussed above in Section III B is trivial in the case of mound growth: When $\alpha \ll \Gamma^{-1}$, the top terrace size becomes comparable to the lattice constant, $L_{\text {top }}=\mathcal{O}(1)$, corresponding to pure statistical Poisson growth 18 .

The fluctuation-dominated regime II with scaling exponents

$$
\gamma^{\prime}=\mu^{\prime}=\frac{i^{*}}{3 i^{*}+2}, \quad \text { (regime II, mounds) }
$$


covers the parameter range $\Gamma^{-1} \ll \alpha \ll \Gamma^{-\delta_{1}}$, where $\delta_{1}=i^{*}\left(2 i^{*}-1\right) /\left(2 i^{*}\left(i^{*}+1\right)+2\right)<1$ for all $i^{*}$. In marked contrast to second layer nucleation, here regime II exists for all $i^{*}$.

For $\Gamma^{-\delta_{1}} \ll \alpha \ll \Gamma^{-\chi / 2}$ nucleation is governed by the exponents of regime III,

$$
\gamma^{\prime}=\frac{i^{*}}{i^{*}+3}, \quad \mu^{\prime}=\frac{i^{*}+1}{i^{*}+3} \quad \text { (regime III, mounds). }
$$

This regime is fluctuation-dominated for $i^{*}<2$, while for $i^{*} \geq 2$ it splits up into fluctuation-dominated and mean field-like subregimes with identical scaling exponents. Both subregimes exist for all $i^{*} \geq 2$, though the fluctuationdominated part becomes very small for large $i^{*}$. For $\alpha>\Gamma^{-\chi / 2}$ one enters the regime of weak step edge barriers, where $l_{\mathrm{ES}}<l_{D}$ and the shape and evolution of the mound morphology are qualitatively different from the wedding-cake regime discussed here [9, 10,17]. A pictorial representation of the scaling regimes is given in Figure 3 .

\section{SUMMARY}

The goal of this paper has been to extend the analysis of [6] to arbitrary critical nucleus sizes, as well as to clarify the relationship between the recent stochastic approaches to second layer nucleation [5, 6, 11] and the earlier mean field theory of Ref. [7]. The essential new ingredient that enters the problem for $i^{*}>1$ is the encounter time $\tau_{\text {enc }}$, which may become larger than the residence time $\tau$. The mean field approach is valid, at least as far as the values of scaling exponents are concerned, when $\tau \ll \tau_{\text {enc }}$, but it fails when $\tau \geq \tau_{\text {enc. }}$. Apart from recovering the results of [11], the analysis has revealed a remarkably simple and universal criterion for fluctuation-dominated second layer nucleation, Eq.(28), which may be useful in situations where nucleation is governed by different mechanisms in the first and second layer. Furthermore I have verified the expectation that fluctuation-dominated nucleation should be more prominent in low dimensionalities, and I have shown that no upper critical nucleus size (beyond which fluctuations can be neglected) exists for nucleation on the top terrace of a mound.

\section{ACKNOWLEDGEMENTS}

I am grateful to S. Heinrichs, P. Maass and T. Michely for useful discussions. This work was supported by DFG within SFB237.

[1] D. Walton, J. Chem. Phys. 37, 2182 (1962).

[2] G. Zinsmeister, Thin Solid Films 2, 497 (1968); ibid. 4, 363 (1969); ibid. 7, 51 (1971).

[3] J.A. Venables, Phil. Mag. 27, 693 (1973); J.A. Venables, G.D.T. Spiller und M. Hanbücken, Rep. Prog. Phys. 47, 399 (1984).

[4] H. Brune, Surf. Sci. Rep. 31, 121 (1998).

[5] J. Rottler and P. Maass, Phys. Rev. Lett. 83, 3490 (1999).

[6] J. Krug, P. Politi and T. Michely, Phys. Rev. B 61, 14037 (2000).

[7] J. Tersoff, A.W. Denier van der Gon and R.M. Tromp, Phys. Rev. Lett. 72, 266 (1994).

[8] I. Elkinani and J. Villain, J. Physique I 4, 949 (1994).

[9] P. Politi and J. Villain, Phys. Rev. B 54, 5114 (1996).

[10] P. Politi, J. Phys. I France 7, 797 (1997).

[11] S. Heinrichs, J. Rottler and P. Maass, Phys. Rev. B (in press).

[12] H. Kallabis, P.L. Krapivsky and D.E. Wolf, Eur. Phys. J. B 5, 801 (1998).

[13] T. Michely, M. Hohage, S. Esch and G. Comsa, Surf. Sci. 349, L89 (1996).

[14] D. Kandel, Phys. Rev. Lett. 78, 499 (1997).

[15] P.L. Krapivsky, Phys. Rev. E 49, 3233 (1994).

[16] A. Pimpinelli, J. Villain and D.E. Wolf, Phys. Rev. Lett. 69, 985 (1992).

[17] For a review see P. Politi, G. Grenet, A. Marty, A. Ponchet and J. Villain, Phys. Rep. 324, 271 (2000).

[18] J. Krug, J. Stat. Phys. 87, 505 (1997).

[19] M. Kalff, P. Šmilauer, G. Comsa and Th. Michely, Surf. Sci. 426, L447 (1999). 


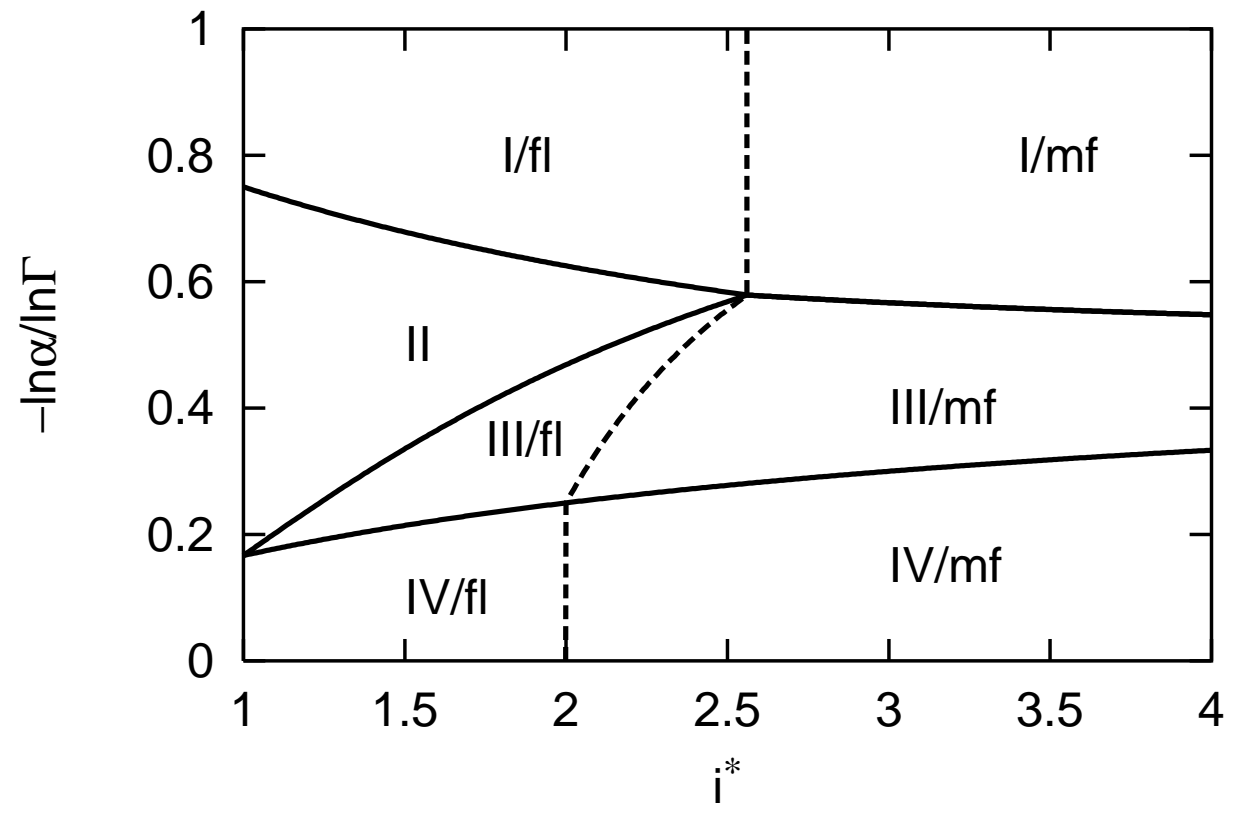

FIG. 1. Scaling regimes for second layer nucleation in two dimensions. Each regime is defined by relations of the form $\Gamma^{-\delta_{k}} \ll \alpha \ll \Gamma^{-\delta_{k^{\prime}}}$, and the full lines show how the boundaries $-\ln \alpha / \ln \Gamma=\delta_{k}$ vary with the critical nucleus size $i^{*}$. The dashed lines indicate transitions between fluctuation-dominated (fl) and mean-field like (mf) subregimes. The explicit expressions for the boundaries are $\delta_{1}=1-3 \chi / 4(\mathrm{I} / \mathrm{fl} \rightarrow \mathrm{II}) ; \delta_{2}=\left(\chi+i^{*}\right)\left(2 i^{*}-1\right) /\left(2 i^{*}\left(i^{*}+1\right)+4\right)(\mathrm{II} \rightarrow \mathrm{III} / \mathrm{fl})$; $\delta_{3}=\left(3 \chi+2 i^{*}-5\right) /\left(2 i^{*}-2\right)(\mathrm{III} / \mathrm{fl} \rightarrow \mathrm{III} / \mathrm{mf}) ; \delta_{4}=\left(5+i^{*}-2 \chi\right) /\left(6+2 i^{*}\right)(\mathrm{I} / \mathrm{mf} \rightarrow \mathrm{III} / \mathrm{mf}) ; \delta_{5}=\chi / 2(\mathrm{III} \rightarrow \mathrm{IV}) . \mathrm{For}$ the island density exponent the conventional expression $\chi=i^{*} /\left(i^{*}+2\right)$ was used. 


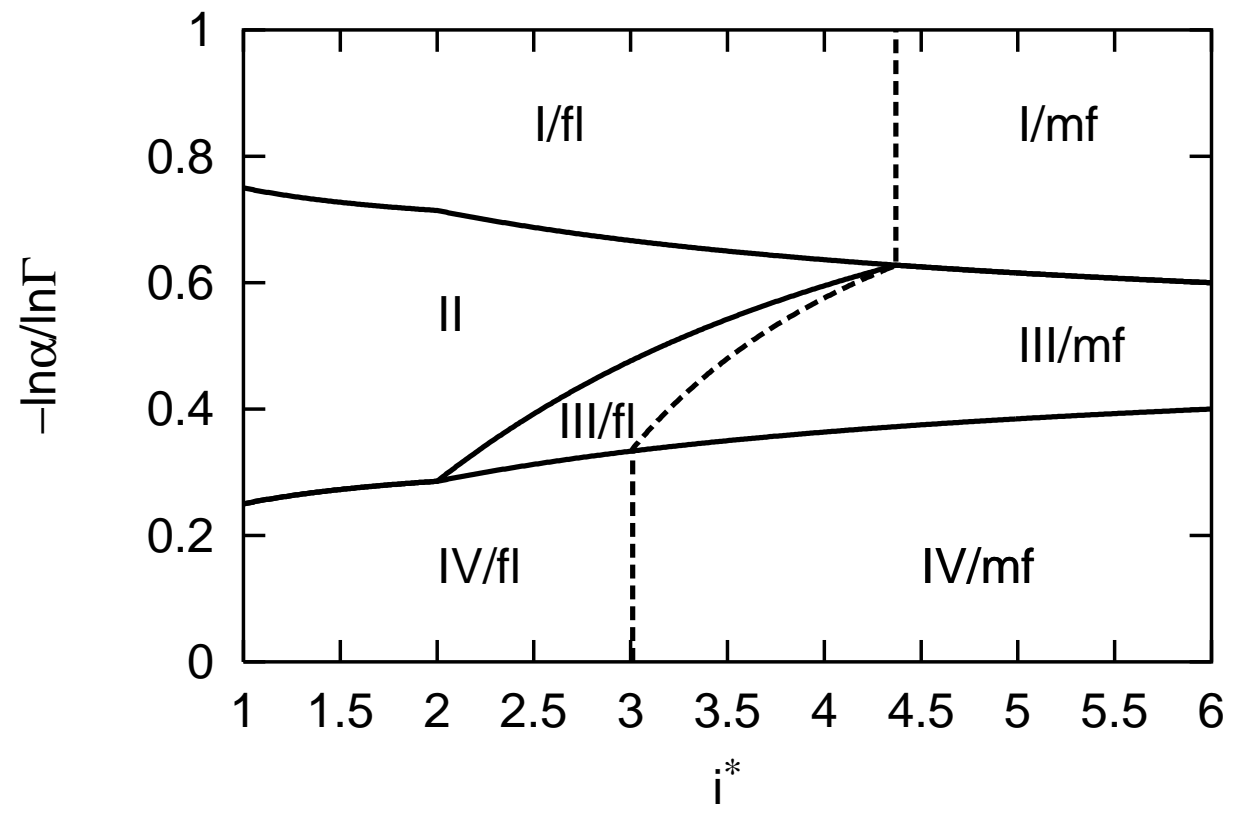

FIG. 2. Scaling regimes for second layer nucleation in one dimension. The explicit expressions for the boundaries are $\delta_{1}=1-\chi(\mathrm{I} / \mathrm{fl} \rightarrow \mathrm{II}$ and $\mathrm{I} / \mathrm{mf} \rightarrow \mathrm{III} / \mathrm{mf}) ; \delta_{2}=\left(\chi+i^{*}\right)\left(i^{*}-1\right) /\left(i^{*}\left(i^{*}+1\right)+2\right)(\mathrm{II} \rightarrow \mathrm{III} / \mathrm{fl}) ; \delta_{3}=1-2(1-\chi) /\left(i^{*}-1\right)(\mathrm{III} / \mathrm{fl}$ $\rightarrow \mathrm{III} / \mathrm{mf}) ; \delta_{4}=\chi(\mathrm{III} \rightarrow \mathrm{IV})$. For the island density exponent $\chi$ the one-dimensional expressions derived in Section IVA $\mathrm{Iere}$ used. 


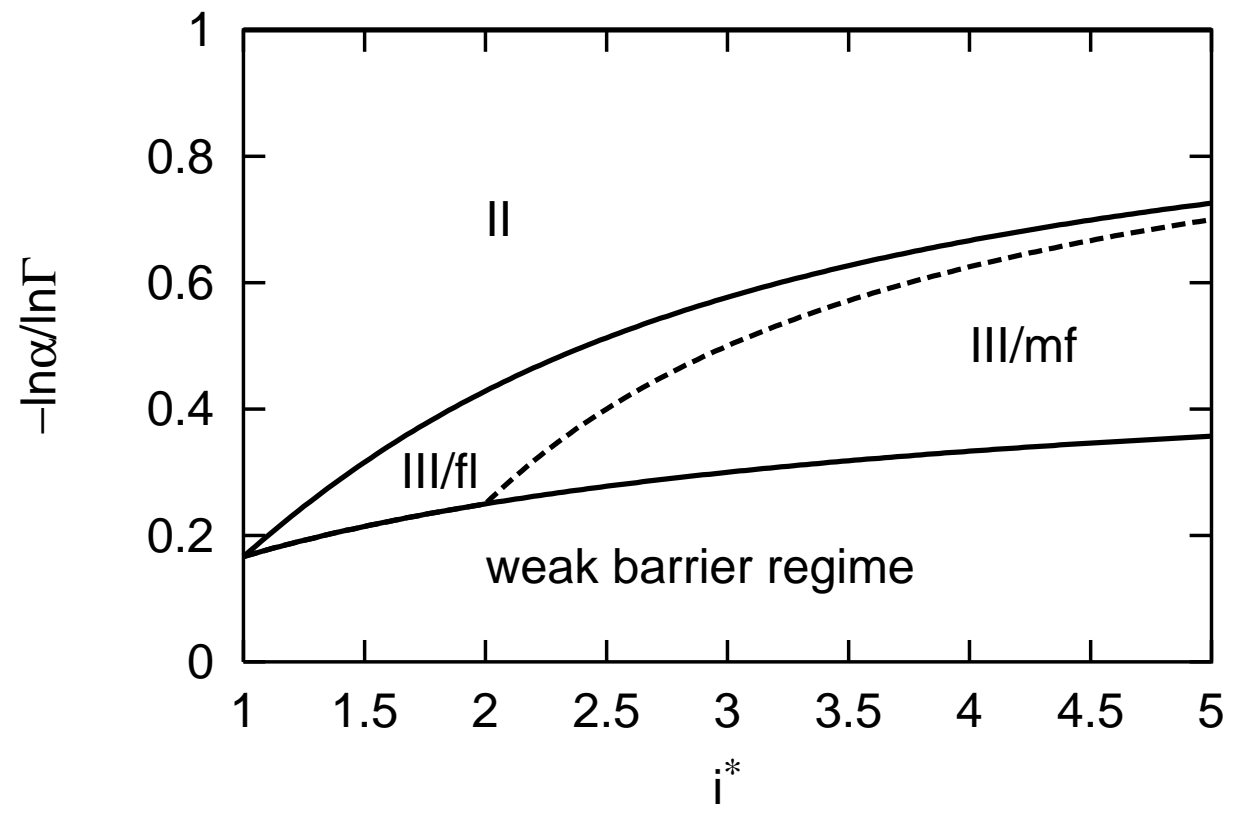

FIG. 3. Scaling regimes for nucleation on top of two-dimensional mounds. The explicit expressions for the boundaries are $\delta_{1}=\left(2 i^{*}-1\right) i^{*} /\left(2 i^{*}\left(i^{*}+1\right)+2\right)(\mathrm{II} \rightarrow \mathrm{III} / \mathrm{fl}) ; \delta_{2}=1-3 / 2 i^{*}(\mathrm{III} / \mathrm{fl} \rightarrow \mathrm{III} / \mathrm{mf}) ; \delta_{3}=\chi / 2(\mathrm{III} \rightarrow \mathrm{IV})$. 